\title{
Control over exciton confinement versus separation in composite films of polyfluorene and CdSe nanocrystals
}

\author{
Y. C. Tseng, M. Tzolov, and E. H. Sargent ${ }^{a)}$ \\ Edward S. Rogers Sr. Department of Electrical and Computer Engineering, University of Toronto, Toronto, \\ M5S 3G4, Canada \\ P. W. Cyr ${ }^{\text {b) }}$ and M. A. Hines ${ }^{\text {b) }}$ \\ Department of Chemistry, University of Toronto, Toronto, M5S 3H6, Canada
}

(Received 26 June 2002; accepted 20 August 2002)

\begin{abstract}
Composite films of polyfluorene derivative poly(9,9-di-(2-ethylhexyl)-fluorenyl-2,7-diyl) and cadmium selenide nanocrystals were investigated using photomodulation spectroscopy exciting only the nanocrystal phase. Efficient charge separation resulting in hole injection into the polymer was observed in films in which the nanocrystals had been stripped of surface trioctylphosphine oxide passivating groups. The resulting induced absorption band centered at $1.2 \mathrm{eV}$ was assigned to bipolarons or $\pi$-dimers formed on the polymer in the near vicinity of the polymer/nanocrystal interface. The intensity dependence of this band suggests bimolecular recombination, supporting the interpretation of the band as due to charge separation. The measured wide distribution of lifetimes for the photogenerated states confirms the glassy nature of the polymer. (C) 2002 American Institute of Physics. [DOI: 10.1063/1.1513214]
\end{abstract}

Semiconductor nanocrystals show promise for use in light-emitting and photovoltaic applications. The ability to tune the emission wavelengths through the quantum size effect, combined with the convenience of solution processability, make nanocrystals attractive for low-cost integrated optoelectronic circuits. When nanocrystals are embedded in a conjugated polymer matrix, the resulting composite system may combine charge injection properties of the polymers with spectrally narrow, size-tunable light-emission behavior of the nanocrystals. Color tunable electroluminescence ${ }^{1}$ and photovoltaic effects ${ }^{2}$ have previously been demonstrated using the composite system poly(paraphenylene vinylene) (PPV)/cadmium selenide ( $\mathrm{CdSe})$.

It is of critical importance to understand alignment of energy bands-quantized conduction and valence band states in the nanocrystals, highest occupied molecular orbital and lowest unoccupied molecular orbital levels in the conjugated polymer-within the composite system. The nature of the heterostructure formed will govern transfer of electrons and holes between nanocrystals and matrix. The composite MEH-PPV/CdSe has been extensively studied by Ginger and Greenham. ${ }^{3}$ The authors reported the separation of photoexcited electron-hole pairs across the boundary of the nanocrystals in this composite resulting in long-lived polarons on the polymer.

Alkylsubstituted polyfluorenes represent another attractive matrix for the nanocrystals owing to the trap-free transport of holes with mobility greater than $3 \times 10^{-4} \mathrm{~cm}^{2} / \mathrm{V} \mathrm{s} ;{ }^{4}$ considerably better thermal stability and photostability than those of the phenylene-vinylene polymers; ${ }^{5}$ and the formation of liquid-crystalline phases $^{6}$ emitting polarized light.

\footnotetext{
a) Author to whom correspondence should be addressed; electronic mail: ted.sargent@utoronto.ca

b) Also at: Edward S. Rogers Sr. Department of Electrical and Computer Engineering, University of Toronto, Toronto, M5S 3G4, Canada.
}

There remains one critical point of uncertainty as to how this polymer class will behave as a matrix for the nanocrystals: since in the PPV/nanocrystal system it is not clear how the polymer and nanocrystals couple on an atomic scale, the different backbone and side chains in polyfluorenes might potentially result in significantly different coupling with the nanocrystals. The charge transfer in the $\mathrm{MEH}-\mathrm{PPV} / \mathrm{CdSe}$ composite has been monitored by detecting photoinduced polaron absorption. ${ }^{3}$ The direct transfer of this experimental approach to polyfluorenes is not obvious because the photoinduced absorption (PA) in this material originates mainly from transitions in the triplet manifold and polaron photogeneration was observed only in thermally cycled films. ${ }^{7}$

It is thus of critical interest to study charge separation at the polyfluorene/nanocrystal interface in order to reveal the nature of the excitations formed by a single charge on a polyfluorene chain.

In this letter, we present evidence of photoinduced charge separation in the composite of polyfluorene derivative poly(9,9-di-(2-ethylhexyl)-fluorenyl-2,7-diyl) (PFE) and $\mathrm{CdSe}$ nanocrystals. We reveal this separation to depend on the presence of trioctylphosphine oxide (TOPO) passivating groups on the surface of the nanocrystals and to result in long-lived states on the polymer.

The polymers PFE (American Dye Sources Inc.) and poly(methyl methacrylate) (PMMA) were used as provided. The CdSe nanocrystals were prepared using the method of Murray et al. ${ }^{8}$ to produce nearly monodisperse TOPOcapped materials. The average size of the nanocrystals was estimated at $3.2 \mathrm{~nm}$ by comparing the solution absorption spectrum with those of known sizes in the literature. ${ }^{8}$ The surface-passivating TOPO ligands were removed by thrice dissolving the isolated $\mathrm{CdSe}$ nanocrystals in a minimal amount of pyridine, precipitating them with hexanes, and centrifuging. This procedure exchanges approximately $90 \%$ of the TOPO ligand groups initially present on the $\mathrm{CdSe}$ 


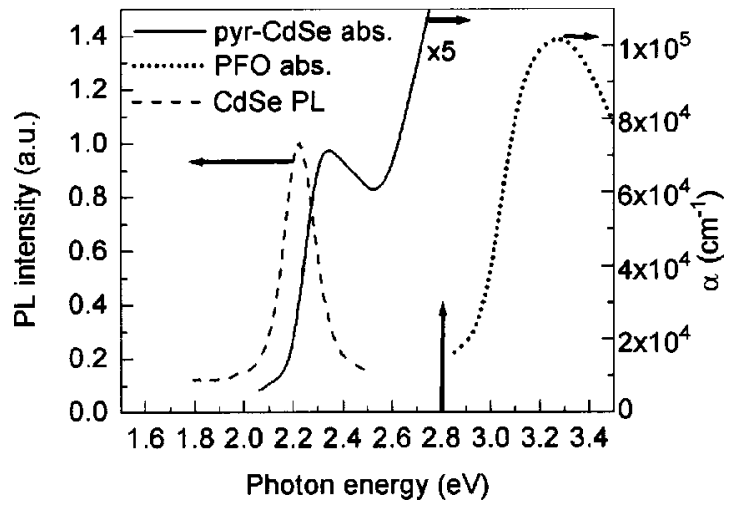

FIG. 1. Absorption spectrum of film of pyr-CdSe (solid line) and PL spectrum of a film of TOPO-capped CdSe nanocrystals (dashed line) together with the absorption of the polyfluorene derivative (dotted line). The arrow at $2.8 \mathrm{eV}$ indicates the photon energy of the pump source. $\alpha$ is the absorption coefficient.

nanocrystal surface ${ }^{9}$ with pyridine. Nanocrystals with exchanged TOPO will be referred to as "pyr-CdSe" throughout this letter. Following the final isolation step, the undried precipitate was dissolved in chloroform, and the concentration of the resulting solution was estimated by evaporating known volumes to dryness and weighing. Composite solutions were prepared with $\sim 75 \mathrm{wt} \%$ CdSe nanocrystal relative to polymer and drop cast onto glass substrates. All results reported in this letter are for films of the abovedescribed composition. We obtained similar results using 50 wt $\%$ films. The thickness of the films studied was estimated to be about several hundred nanometers.

The photomodulation experiments were performed using the $442 \mathrm{~nm}$ line of a HeCd laser as a pump beam and a tungsten halogen lamp as a probe beam. The probe beam was spectrally analyzed by a single grating monochromator Triax 320. Detection was accomplished with Si (430-1100 nm) and InGaAs $(930-1650 \mathrm{~nm})$ photodiodes connected to a current-to-voltage preamplifier and a lock-in amplifier SR830. All measurements were made under vacuum in a closed-cycle He cryostat with quartz optical ports. The data presented herein were taken at room temperature; lowtemperature measurements are only briefly mentioned. The absorption data were measured using a Cary 500 spectrophotometer and the photoluminescence (PL) spectra using a PTI spectrofluorometer QM-2000-7, each at room temperature.

Excitation of PFE films in the high absorption region $(3.3 \mathrm{eV})$ gives very bright PL having the first- and secondvibronic maximum at 2.95 and $2.82 \mathrm{eV}$ at room temperature. The excitation at $2.805 \mathrm{eV}(442 \mathrm{~nm})$ is within the optical gap of PFE (Fig. 1) and, therefore, should excite the glassy films of PFE minimally. We were unable to detect any PL or PA from the PFE films in the case of excitation at $442 \mathrm{~nm}$ in agreement with previous studies. ${ }^{10}$

At the same time, $442 \mathrm{~nm}$ light is strongly absorbed in the nanocrystals. We presume on this basis that photogeneration of charge carriers in the composite material occurs overwhelmingly in the nanocrystals.

The nanocrystals have been characterized by absorption and PL (excitation at $370 \mathrm{~nm}$ ) spectroscopy as shown in Fig. 1. The Stokes shift of the PL maximum as well as the width of the PL band are typical for CdSe nanocrystals. ${ }^{11}$ The PL Downloaded 26 Nov 2002 to 128.100.138.68. Redistribution subject

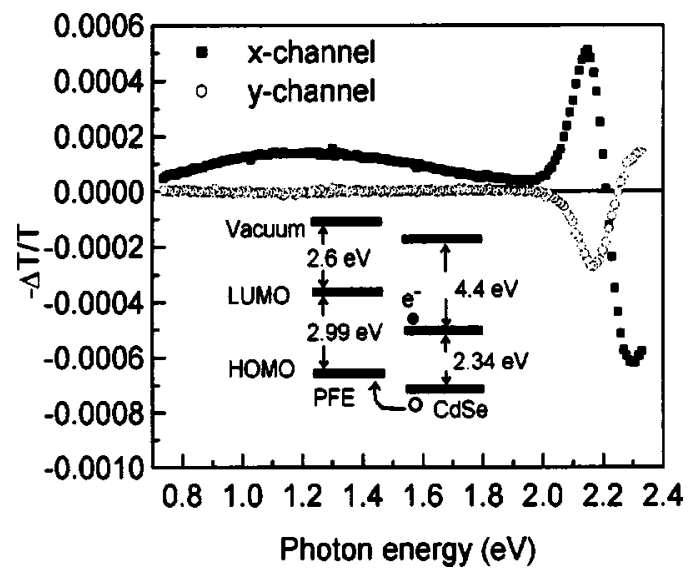

FIG. 2. PA spectrum of pyridine-capped CdSe nanocrystals embedded in PFE. The in-phase signal (solid squares) was obtained after numerical rotation of the phase which zeroed the quadrature signal (open circles) at about $1.2 \mathrm{eV}$. The inset contains a schematic representation of the relative position of the energy levels in the composite material PFE/pyr-CdSe.

spectrum pictured in Fig. 1 was obtained using CdSe capped with surface-passivating TOPO ligands due to the fact that the nanocrystals treated with pyridine exhibit dramatically decreased PL.

The photomodulation spectrum of the PFE/pyr-CdSe film is characterized by a broad PA band peaking at about 1.2 eV (Fig. 2). The control samples of PMMA/pyr-CdSe and $\mathrm{PFE} / \mathrm{CdSe}$ do not show this band. We therefore assign it to the presence of charged excitations on the PFE as a result of charge transfer between the nanocrystals and the conjugated polymer facilitated by the removal of the surface passivating TOPO ligands. This process can be explained with reference to the simple band scheme shown in the inset of Fig. 2. The electron affinity of the nanocrystals is taken from the bulk value. The band gap energies are extracted from the data in Fig. 1. The value for the CdSe nanocrystals of $E_{\mathrm{CdSe}}$ $=2.34 \mathrm{eV}$ was taken at the maximum of the absorption peak and the value for the polymer of $E_{\mathrm{PFE}}=2.99 \mathrm{eV}$ was calculated using the formula for a one-dimensional semiconductor, ${ }^{12}$

$$
\alpha h \nu \sim\left(h \nu-E_{g}\right)^{1 / 2},
$$

where $\alpha$ is the absorption coefficient, $E_{g}$ is the bandgap energy, and $h \nu$ is the incident photon energy.

The excitation of an electron-hole pair within the nanocrystals is followed by the transfer of the hole onto the polymer while the electron remains on the nanocrystal. The presence of charges on the chain of a conjugated polymer leads to the appearance of new states within the optical gap. ${ }^{13}$ For the case of poly(9,9-dioctylfluorene), Cadby et al. ${ }^{7,10}$ have reported the presence of polaron and bipolaron states in thermally cycled films. The band in Fig. 2 cannot be identified with the polaron states observed in Refs. 7 and 10 because of the large difference in the energy position and in the width of the band. The bipolaron band has been detected in the spin $1 / 2 \lambda$-absorption detected magnetic resonance ${ }^{7}$ and the energy position and width are closer to the band observed in the composite material in Fig. 2. Another possibility is the formation of $\pi$-dimers representing the coupling between polarons at different chains. Bipolarons and $\pi$-dimers are expected to be formed in heavily doped samples. In the case of to AlP license or copyright, see http://ojps.aip.org/aplo/aplcr.jsp 


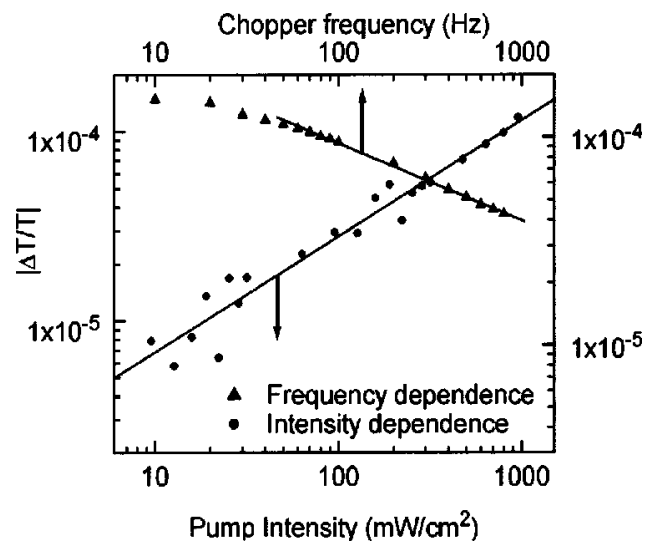

FIG. 3. Frequency and pump intensity dependence of the magnitude of the band at $1.2 \mathrm{eV}$ for $955 \mathrm{~mW} / \mathrm{cm}^{2}$ pump beam intensity and $40 \mathrm{~Hz}$, respectively. The lines serve only as a guide.

the excitation of the nanocrystals followed by injection of holes into the polymer, the volume in which the injected holes reside is restricted near the polymer/nanocrystal interface. The coupling between separate charges is highly probable in this restricted space, as in the case of heavy doping, and the formation of bipolarons or $\pi$-dimers can occur. A precise assignment of the observed band requires information about the spin state of the photoexcited states.

Our results point to the essential role of the removal of the surface passivating TOPO groups in order for charge transfer to occur. The ligands, when present, prevent the nanocrystals from approaching the PFE chains sufficiently close for effective charge separation to occur. Similar arguments were presented by Greenham et al. ${ }^{2}$ and experimentally confirmed for the case of PPV/CdSe composites. ${ }^{3}$

Figure 2 shows the presence of photoinduced bleaching and absorption bands observed in the spectral range $h \nu$ $>2 \mathrm{eV}$ which were present in all samples. We attribute these bands to the bleaching of the ground state of the nanocrystal ${ }^{14}$ combined with temperature modulation enhanced by the poor thermal conductivity of the glass substrate. In addition, these bands' phase is different from that at $1.2 \mathrm{eV}$. The fact that numerical phase rotation (Fig. 2) can not make zero the out-of-phase component in the spectral range $2.1-2.5 \mathrm{eV}$ at the same angle of rotation as it does for the $1.2 \mathrm{eV}$ band indicates contributions to these signals from different sources.

The frequency dependence of the band at $1.2 \mathrm{eV}$ presented in Fig. 3 cannot be fit using a single lifetime. The slope of the curve $|\Delta T / T| \sim f^{-n}$ varies between different samples with $n$ in the range $0.36-0.25$, and the graph suggests a distribution of lifetimes in the millisecond range. Over the chopping frequency range presented, there occurs no slope change to $n=1$, indicating that states exist with lifetimes shorter than $0.5 \mathrm{~ms}$. The absence of a definite lifetime of the photoexcitations is consistent with the amorphous, glassy nature of the polymer and the possible distortions of the polymer chains around the nanocrystals where most of the separated charges reside. The large width of the PA band peaking at $1.2 \mathrm{eV}$ in Fig. 2 can be interpreted as another manifestation of the disorder in the films.

The sublinear intensity dependence of the PA shown in Fig. $3\left(|\Delta T / T| \sim I^{-0.61}\right)$ suggests that the recombination is Downloaded 26 Nov 2002 to 128.100 .138 .68 . Redistribution subject bimolecular. For bimolecular recombination, ${ }^{3}$ the intensity dependence in the steady state goes as the square root, however, for modulation frequencies higher than the inverse lifetime of the state, the dependence is linear. In the presence of states with different lifetimes, the intensity dependence will lie between the square root and linear and the exact value will depend on the modulation frequency used during the measurements. The measured value of 0.61 suggests that the recombination is bimolecular with states with different lifetimes. The bimolecular recombination mechanism supports the attribution of the band at $1.2 \mathrm{eV}$ to separated charge carriers.

The PA shows little temperature dependence. The amplitude of the signal at $1.2 \mathrm{eV}$ increases about three times from room temperature to $10 \mathrm{~K}$ accompanied by a phase shift indicating an increase in the lifetime under cooling.

The experimental results point to the conclusion that interfacial charge separation occurs in the PFE/pyr-CdSe composite upon illumination. The efficient coupling between the two materials is inhibited by the presence of TOPO passivating groups on the surface of the nanocrystal. The injection of holes into the conjugated polymer after the photoexcitation of the nanocrystals allowed us to detect charged excitations in the glassy PFE. Most probably, they form pairs on one or on separate chains and the corresponding electronic levels give rise to a broad absorption band with a maximum at 1.2 $\mathrm{eV}$. Because of the glassy nature of the polymer, the lifetime and energy of the photogenerated states are site dependent. The findings in this letter suggest the PFE/CdSe composite may provide improved efficiency in photovoltaic applications due to the good transport properties of PFE.

The authors thank Professor Gregory D. Scholes for the use of his facilities in the synthesis of the nanocrystals and C. Paquet for kindly providing the PMMA used in this experiment.

${ }^{1}$ V. L. Colvin, M. C. Schlamp, and A. P. Alivisatos, Nature (London) 370, 354 (1994).

${ }^{2}$ N. C. Greenham, X. Peng, and A. P. Alivisatos, Phys. Rev. B 54, 17628 (1996).

${ }^{3}$ D. S. Ginger and N. C. Greenham, Phys. Rev. B 59, 10622 (1999).

${ }^{4}$ M. Redecker, D. D. C. Bradley, M. T. Bernius, M. Inbasekaran, W. W. Wu, and E. P. Woo, Appl. Phys. Lett. 73, 629 (1998).

${ }^{5}$ M. Kreyenschmidt, G. Klaerner, G. Fuhrer, J. Ashenhurst, S. Karg, W. D. Chen, V. Y. Lee, J. C. Scott, and R. D. Miller, Macromolecules 31, 1099 (1998).

${ }^{6}$ M. Grell, D. D. C. Bradley, G. Ungar, J. Hill, and K. S. Whitehead, Macromolecules 32, 5810 (1999).

${ }^{7}$ A. J. Cadby, P. A. Lane, M. Wohlgenannt, C. An, Z. V. Vardeny, and D. D. C. Bradley, Synth. Met. 111, 515 (2000).

${ }^{8}$ C. B. Murray, D. J. Norris, and M. G. Bawendi, J. Am. Chem. Soc. 115, 8706 (1993).

${ }^{9}$ J. E. Bowen Katari, V. L. Colvin, and A. P. Alivisatos, J. Phys. Chem. 98, 4109 (1994).

${ }^{10}$ A. J. Cadby, P. A. Lane, H. Mellor, S. J. Martin, M. Grell, C. Giebeler, D. D. C. Bradley, M. Wohlgenannt, C. An, and Z. V. Vardeny, Phys. Rev. B 62, 15604 (2000).

${ }^{11}$ M. G. Bawendi, P. J. Carroll, W. L. Wilson, and L. E. Brus, J. Chem. Phys. 96, 946 (1992).

${ }^{12}$ N. Kirova, S. Brazovskii, and A. R. Bishop, Synth. Met. 100, 29 (1999).

${ }^{13}$ Z. V. Vardeny and X. Wei, in Handbook of Conducting Polymers, edited by T. A. Skotheim, R. L. Elsenbaumer, and H. R. Reynolds (Marcel Dekker, New York, 1998), p. 639.

${ }^{14}$ D. S. Ginger, A. S. Dhoot, C. E. Finlayson, and N. C. Greenham, Appl. Phys. Lett. 77, 2816 (2000).

to AIP license or copyright, see http://ojps.aip.org/aplo/aplcr.jsp 